\title{
Influence of an Adenosine Deaminase Inhibitor, Erythro-9-(2-hydroxy-3- nonyl) Adenine Hydrochloride, on 5-HT2CR mRNA Editing in Primary Cultured Cortical Cells
}

\author{
Pham Nguyet Thi Hang, ${ }^{a}$ Michihisa Tohda, ${ }^{*, a}$ Yasuhiro Tezuka, ${ }^{b}$ and Kinzo Matsumoto ${ }^{a}$ \\ ${ }^{a}$ Division of Medicinal Pharmacology, Institute of Natural Medicine, University of Toyama; and ${ }^{b}$ Division of Natural \\ Products Chemistry, Institute of Natural Medicine, University of Toyama; Sugitani 2630, Toyama 930-0194, Japan. \\ Received October 15, 2009; accepted December 4, 2009; published online December 24, 2009
}

\begin{abstract}
Treatment of primary cultured cortical cells with erythro-9-(2-hydroxy-3-nonyl) adenine hydrochloride (EHNA), an inhibitor of adenosine deaminase (ADAR), for $6 \mathrm{~d}$ significantly and concentration-dependently reduced the editing efficacy at sites $C$ and $D$ but not at site A or B of 5-HT2CR mRNA. The treatment failed to affect the editing of ADAR-2 pre-mRNA and a subunit of the $\alpha$-amino-3-hydroxy-5-methyl-4-isoxazolepropionate (AMPA)-type glutamate receptor (GluR2) mRNA. These findings suggest that EHNA is useful for clarifying the functional roles of 5-HT2CR mRNA editing at sites $C$ and D.
\end{abstract}

Key words 5 -HT2C receptor mRNA editing; adenosine deaminase; psychiatric disorder

The adenosine to inosine RNA editing process mediated by adenosine deaminase acting on RNA (ADARs) has been reported to play critical roles in the functional development of the central nervous system by modulating the functions of neurotransmitter receptors. Serotonin 2C receptor (5HT2CR) mRNA undergoes editing by these ADARs at $5 \mathrm{nu}-$ cleotide positions (sites $\mathrm{A}-\mathrm{E}$ ) located in the sequence encoding the second intracellular loop of 5-HT2CR. This editing allows for the generation of 32 mRNA variants and 24 protein isoforms of the receptor differing in G-protein coupling efficiency. ${ }^{1)}$ The involvement of 5-HT2CR mRNA editing in psychiatric disorders has been hypothesized based on etiological and pharmacological studies. Altered editing of 5-HT2CR mRNA was detected in the postmortem brains of patients with major depression, schizophrenia and bipolar disorder. $^{2-4)}$ Yang et al. ${ }^{5}$ found that 5-HT2CR mRNA editing frequency in human glioblastoma cell lines was altered by treatment with interferon which causes depression as an adverse effect. These findings also raised the possibility that compounds with the ability to inhibit/stimulate the editing of 5-HT2CR mRNA are useful for clarifying the role of 5-HT2CR mRNA editing in psychiatric disorders.

Our previous studies have shown that the editing efficacy of 5-HT2CR mRNA and ADAR-2 pre-mRNA and the level of ADAR-2 pre-mRNA are altered during the development of the brain in rats. ${ }^{6,7)}$ These results suggested that 5-HT2CR mRNA editing and these enzymes play a role in the brain's maturation. To find a pharmacological tool with which to investigate the roles of 5-HT2CR mRNA editing, we examined the effect of erythro-9-(2-hydroxy-3-nonyl) adenine hydrochloride (EHNA), a well-known inhibitor of adenosine deaminase (ADA) which breaks extracellular adenosine, ${ }^{8)}$ on the editing of 5-HT2CR mRNA at sites A-D in cultured cortical cells.

\section{MATERIALS AND METHODS}

Cultured cortical cells prepared from E20 embryos as described previously ${ }^{6,7)}$ were used for the experiments. The cells were exposed to vehicle (control) or EHNA included in culture media at concentrations of 30 and $60 \mu \mathrm{M}$ for $6 \mathrm{~d}$.
After a 6-d cultivation period, total RNA was extracted, and RT-PCR was carried out as described previously. ${ }^{6,7)}$ The specific primers for target genes to identify the bases at editing sites were: 5-HT2CR mRNA (NM_008312); 5'-atg tcc cta gec att get gat atg ctg gtg-3' (sense), 5' -atg cca cga agg acc cga tga gaa cga agt-3' (antisense), and Texas Red-labeled: $5^{\prime}$-ata ttt gtg ccc cgt cgt ga; ADAR-2 pre-mRNA (NW_047598), 5' -atg tgc tgg tga act cac ag-3' (sense), 5' -gtg gtg ccc aga aag agt gg-3' (antisense) and Texas Red-labeled: 5'-taa gta gga gag gca ata ggt ccg-3'; and GluR2 (NM_017261), 5'-tat atg agg agt gca gag cc-3' (sense), 5' ata ctc cag caa cgt tgc tc-3' (antisense) and Texas Red-labeled: $5^{\prime}$-ctg tgt ttg tga gga cta cc-3'. Based on pilot studies where we ran specific primers for target genes, the proper cycle number for the amplification of each target gene was chosen (5-HT2CR mRNA including the editing sites: 35; ADAR-2 pre-mRNA: 28 cycles; GluR2: 32 cycles). To analyze the editing frequencies at sites $\mathrm{A}-\mathrm{D}$ of 5 -HT2CR mRNA, a direct sequencing method with a Tex-Red-labeled primer adapted to the PCR product was employed as previously reported. ${ }^{6,7)}$

All data were expressed as the mean \pm S.E.M. and analyzed by a one-way analysis of variance (ANOVA) followed by the Student-Newman-Keuls test. Differences of $p<0.05$ were considered significant.

\section{RESULTS}

We previously demonstrated that the editing frequencies of 5-HT2CR mRNA reached maximal levels at days 6-9 in primary cultured cortical cells. ${ }^{7)}$ Therefore, in this study, the primary cultured cortical cells were treated with different concentrations of EHNA $(10-100 \mu \mathrm{M})$ for $6 \mathrm{~d}$. Based on a preliminary study, we evaluated the effect of 30 and $60 \mu \mathrm{M}$ EHNA on the editing frequencies at sites A-D of 5-HT2CR mRNA in the cultured cells. Microscopic observation revealed an enhancement of neurite outgrowth during the 6-d cultivation. Treatment of the cells with EHNA for $6 \mathrm{~d}$ did not cause any morphological changes (Fig. 1-1)). Meanwhile, as illustrated in Fig. 1-2)), EHNA treatment $(30-60 \mu \mathrm{M})$ concentration-dependently decreased the editing frequencies at 
1)

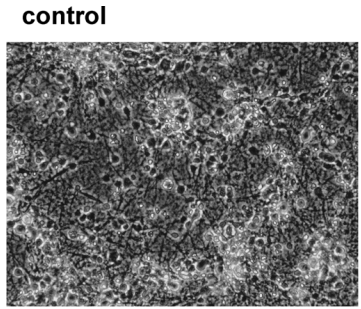

$30 \mu$ M EHNA

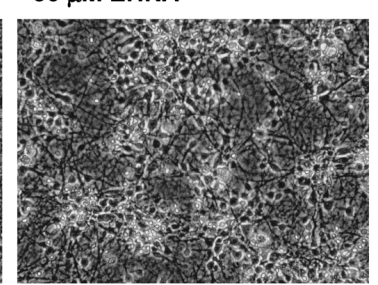

$60 \mu \mathrm{M}$ EHNA

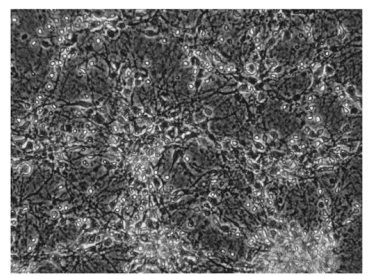

2)
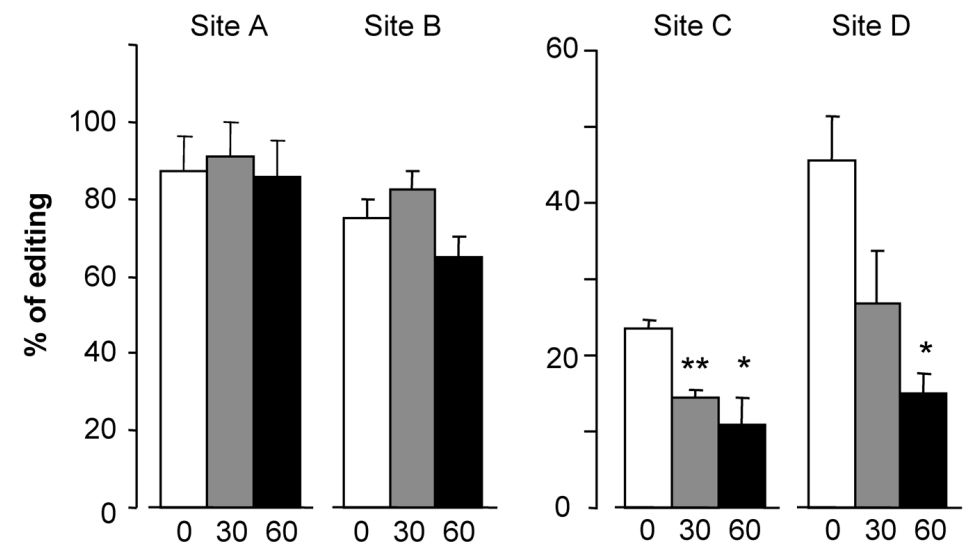

EHNA $(\mu M)$

${ }^{* *} \mathrm{P}<0.01, \quad{ }^{*} \mathrm{P}<0.05$ vs control group $(\mathrm{n}=3)$

Fig. 1. The Effects of EHNA Treatment on Editing of 5-HT2CR mRNA in Primary Cultured Rat Cortical Cells

Cortical cells were prepared from rat brains at embryonic day 20 (E20) and cultured in the presence and absence of 30 and $60 \mu \mathrm{M}$ EHNA, an inhibitor of ADARs, for $6 \mathrm{~d}$. 1) Typical morphology of primary cultured cortical cells treated with 30 and $60 \mu \mathrm{m}$ EHNA for $6 \mathrm{~d}$. 2) EHNA-induced changes in editing frequencies at sites A-D of 5-HT2CR mRNA. The extraction of RNA from the cultured cells, RT-PCR, and analysis of PCR-products were conducted as described in the text. Editing at site E was not detected in cultured cortical cells at day 6 of cultivation. Each value shows the mean \pm S.E.M. obtained from 3 independent experiments. $* p<0.05$ and $* * p<0.01 v s$. control group.

Table 1. Editing Levels of GluR2 mRNA and ADAR-2 Pre-mRNA in Cultured Cortical Cells with or without EHNA Treatment

\begin{tabular}{cccc}
\hline \hline & \multicolumn{3}{c}{ Editing efficacy (\%) } \\
\cline { 2 - 4 } Editing sites & $\begin{array}{c}\text { Control } \\
(n=4)\end{array}$ & $\begin{array}{c}\text { EHNA }(30 \mu \mathrm{M}) \\
(n=4)\end{array}$ & $\begin{array}{c}\text { EHNA }(60 \mu \mathrm{M}) \\
(n=5)\end{array}$ \\
\hline ADAR-2 pre mRNA & & & \\
-2 & $5.9 \pm 3.9$ & $7.6 \pm 5.3$ & $12.7 \pm 1.8$ \\
-1 & $23.5 \pm 2.9$ & $27.6 \pm 5.1$ & $30.7 \pm 1.1$ \\
10 & $18.7 \pm 2.5$ & $17.5 \pm 0.8$ & $15.2 \pm 1.2$ \\
14 & $33.8 \pm 2.2$ & $33.5 \pm 0.8$ & $31.9 \pm 2.4$ \\
23 & 0 & $5.1 \pm 5.1$ & 0 \\
24 & $30.1 \pm 8.8$ & $33.7 \pm 3.9$ & $38.6 \pm 3.8$ \\
GluR2 mRNA & & & \\
Site R/G & $42.3 \pm 2.4$ & $50.5 \pm 2.7$ & $55.1 \pm 3.6$ \\
Site Q/R & $100 \pm 0$ & $100 \pm 0$ & $100 \pm 0$ \\
\hline
\end{tabular}

RT-PCR was conducted using a specific primer set to include the editing sites of GluR2 mRNA and ADAR-2 pre-mRNA. The PCR products including editing sites were analyzed using Texas red-labeled primers. The GluR2 mRNA contained 2 sites: sites $\mathrm{Q} / \mathrm{R}$ and $\mathrm{R} / \mathrm{G}$. The ADAR-2 pre-mRNA contained six positions: $-2,-1,+10$ $+14,+23$ and +24 . Each value represents the mean \pm S.E.M. The number of dishes used in the experiments is indicated in parentheses.

sites C and D: a reduction from 23 to $10.5 \%$ and from 44.7 to $14.8 \%$ at $60 \mu \mathrm{M}$ of EHNA, respectively. This treatment had no effect on the editing frequency at site A or B. To clarify whether the effect of EHNA on mRNA editing is specific to sites C and D of 5-HT2CR mRNA, we also analyzed the effects of EHNA on the editing of genes encoding ADAR-1 and -2 and GluR2 in cultured cortical cells because ADAR-1 and -2 are also responsible for RNA editing. ${ }^{9}$ EHNA treatment $(30-60 \mu \mathrm{M})$ of the cells for $6 \mathrm{~d}$ had no effect on the editing frequencies at sites $\mathrm{Q} / \mathrm{R}$ and $\mathrm{R} / \mathrm{G}$ of GluR2 mRNA, or at the six positions of ADAR-2 pre-mRNA (Table 1).

\section{DISCUSSION}

This study aimed to find a pharmacological tool to investigate the physiological roles of 5-HT2CR mRNA editing which is thought to be involved in psychiatric disorders. To this end, we examined the effects of EHNA, an inhibitor of ADA, on the editing in cultured cortical cells. EHNA (30$60 \mu \mathrm{M})$ preferentially inhibited editing at sites $\mathrm{C}$ and $\mathrm{D}$ of 5-HT2CR mRNA without affecting that at sites A and B or the editing of other genes. It is likely that EHNA is a specific/preferential inhibitor for the enzyme involved in editing at sites C/D of 5-HT2CR mRNA. Since sites C/D are edited by ADAR-2, whereas sites A/B are edited by ADAR-1, EHNA seems to be specific to ADAR-2. In contrast to its effect on the editing of 5-HT2CR mRNA, EHNA had no effect on the editing of GluR2 mRNA. Previous studies ${ }^{10,11)}$ have demonstrated that the $\mathrm{Q} / \mathrm{R}$ site of GluR2 mRNA is predominantly edited by ADAR-2, whereas the $R / G$ site is edited by both ADAR-1 and -2. EHNA treatment inhibited the editing at sites $\mathrm{C}$ and $\mathrm{D}$ but not that at the $\mathrm{Q} / \mathrm{R}$ site, although these sites are reportedly edited by ADAR-2. These results may indicate that the effects of EHNA depend on the characteristics of the site of editing in addition to selectivity forward ADAR-2. The $Q / R$ site is usually edited at a rate of approximately $100 \%$ in adult brain as in this report. A slight decrease in editing frequency at the $\mathrm{Q} / \mathrm{R}$ site $(62-100 \%)$ resulting in abnormal permeation by $\mathrm{Ca}^{2+}$ has been observed in ALS (amyotrophic lateral sclerosis) patients. ${ }^{12)}$ Namely, since the $\mathrm{Q} / \mathrm{R}$ site must be highly edited for the physiological 
function, the sensitivity of this site to ADAR-2 activity in itself may be relatively high. Thus, the inhibitor EHNA would not be able to contribute to inhibit this editing. On the other hand, as the editing rate of sites $\mathrm{C}$ and $\mathrm{D}$ is maintained at a low level (Fig. 1), these sites easily to accept the deamination activity controlled by ADAR-2. In other words, sites C and D are affected by ADAR-2 inhibitor EHNA. Changes in editing frequency may not be simply controlled by ADARs alone. Phosphorylation may also be involved in the changes in frequency of RNA editing. Further clarification of related factors is necessary to know the functional meaning of RNA editing. Changes in editing frequencies at sites $C$ and D of 5HT2CR mRNA have been reported to be involved in psychiatric disorders in humans ${ }^{1)}$ and to be observed in animal models of psychiatric disorders such as depression ${ }^{13)}$ and anxiety. ${ }^{14)}$ Furthermore, treatment of mice with an antidepressant, fluoxetine, decreased the editing frequency at site C. ${ }^{15)}$ These reports suggested a close linkage between 5HT2CR mRNA editing and psychiatric disorders. The present findings about high sensitivity of EHNA against 5HT2CR mRNA editing at sites C and D suggest that EHNA is useful to investigate the functional roles of these sites' mRNA editing such as the generation of short variant mRNA. ${ }^{16,17)}$

\section{REFERENCES}

1) Burns C. M., Chu H., Rueter S. M., Hutchinson L. K., Canton H., Sanders-Bush E., Emeson R. B., Nature (London), 387, 303-308
(1997).

2) Gurevich I, Tamir H., Arango V., Dwork A. J., Mann J. J., Schmauss C., Neuron, 34, 349-356 (2002).

3) Iwamoto K., Kato T., Neurosci. Lett., 346, 169-172 (2003).

4) Tohda M., Nomura M., Nomura Y., J. Pharmacol. Sci., 100, 427-432 (2006).

5) Yang W., Wang Q., Kanes S. J., Murray J. M., Nishikura K., Brain Res. Mol. Brain Res., 124, 70-78 (2004).

6) Hang P. N., Tohda M., Matsumoto K., Neurosci. Res., 36, 398-403 (2008).

7) Tohda M., Hang P. N., Matsumoto K., Biol. Pharm. Bull., 32, 289292 (2009).

8) Cristalli G., Eleuteri A., Volpini R., Vittori S., Camaioni E., Lupidi G., J. Med. Chem., 37, 201-205 (1994).

9) Higuchi M., Maas S., Single F. N., Hartner J., Rozov A., Burnasjev N., Feldmeyer D., Sprengel R., Seeburg P. H., Nature (London), 406, 78 81 (2000).

10) Melcher T., Maas S., Herb A., Sprengel R., Seeburg P. H., Higuchi M., Nature (London), 379, 460-464 (1996).

11) Wang Q., Khillan J., Gadue P., Nishikura K., Science, 290, 1765 1768 (2000).

12) Maas S., Kawahara Y., Tamburro K. M., Nishikura K., RNA Biol., 3, $1-9$ (2006).

13) Iwamoto K., Nakatani N., Bundo M., Yoshikawa T., Katol T., Neurosci. Res., 53, 69-76 (2005).

14) Hackler E. A., Airey D. C., Shannon C. C., Sodhi M. S., Sanders-Bush E., Neurosci. Res., 55, 96-104 (2006).

15) Gurevich I., Englander M. T., Adlersberg M., Siegal N. B., Schmauss C., J. Neurosci., 22, 10529-10532 (2002).

16) Tohda M., Sukma M., Nomura Y., Watanabe H., Jpn. J. Pharmacol., 90, 138-144 (2002).

17) Tohda M., Sukma M., Watanabe H., J. Pharmacol. Sci., 96, 164-169 (2004). 